\title{
Low-energy electron microscopy observations of GaN homoepitaxy using a supersonic jet source
}

\author{
A. Pavlovska, V. M. Torres, E. Bauer, R. B. Doak, and I. S. T. Tsong ${ }^{\text {a) }}$ \\ Department of Physics and Astronomy, Arizona State University, Tempe, Arizona 85287-1504 \\ D. B. Thomson and R. F. Davis \\ Department of Materials Science and Engineering, North Carolina State University, Raleigh, \\ North Carolina 27695-7907
}

(Received 22 February 1999; accepted for publication 24 June 1999)

\begin{abstract}
A study of the homoepitaxial growth of $\mathrm{GaN}(0001)$ layers was conducted in situ and in real time using the low-energy electron microscope. The Ga flux was supplied by an evaporative cell while the $\mathrm{NH}_{3}$ flux was supplied via a seeded-beam supersonic jet source. At growth temperatures of $665^{\circ} \mathrm{C}$ and $677^{\circ} \mathrm{C}$, smooth $\mathrm{GaN}(0001)$ layers with well-defined step structures were grown on $\mathrm{GaN}(0001)$ substrates prepared by metalorganic chemical vapor deposition. In general, nonfaceted homoepitaxial layers were achieved when the $\mathrm{Ga} / \mathrm{NH}_{3}$ flux ratios exceeded 2, starting with a Ga-covered substrate surface, in the temperature range of $655-710^{\circ} \mathrm{C}$. (C) 1999 American Institute of Physics. [S0003-6951(99)04533-7]
\end{abstract}

The selected energy epitaxy (SEE) approach of GaN growth is based on a concept demonstrated by $\mathrm{Ceyer}^{1,2}$ that upon collision with a surface, a reactant can overcome the potential barrier to dissociative chemisorption by its translational energy. In this case, the reactant molecules, $\mathrm{NH}_{3}$, are seeded into a beam of $\mathrm{He}$ atoms from a supersonic jet (SSJ) source. We have recently shown that with the SSJ, essentially monoenergetic beams of $\mathrm{NH}_{3}$ in the energy range of $0.20-0.65 \mathrm{eV}$ with a full width at half maximum of $0.1 \mathrm{eV}$ can be easily produced. ${ }^{3-5}$ Such an approach has the advantage of fine tuning an energy window in which dissociation and reaction are promoted, surface diffusion is enhanced, while defect creation due to energetic collisions is minimized. All of these processes can, in principle, take place at a lower growth temperature since part of the translational energy of the flux species is converted into potential energy to overcome the activation barrier while the rest goes to promote mobility of the adsorbed species to migrate to preferred sites such as step edges on the surface. Since the activation barrier for dissociation and chemisorption of $\mathrm{NH}_{3}$ on a $\mathrm{GaN}(0001)$ surface has been calculated to have an upper limit of $0.5 \mathrm{eV},{ }^{6}$ and experimentally determined to be 0.25 $\pm 0.1 \mathrm{eV},{ }^{5}$ the SSJ is ideally suited for low-temperature GaN growth. A review of the epitaxy of group III nitrides by SSJ has been given recently by Ferguson and Mullins. ${ }^{7}$ In this letter, we describe in situ real-time observations of GaN homoepitaxy by SSJ using a low-energy electron microscope (LEEM). ${ }^{8}$ The experimental parameters, i.e., substrate preparation, growth temperature, and flux ratio, leading to smooth basal plane (0001) growth, were explored and determined in our LEEM studies.

The experimental configuration for conducting the LEEM observations of GaN growth by SSJ has been described in detail previously. ${ }^{4}$ The base pressure in the LEEM was $\sim 1 \times 10^{-10}$ Torr, which increased to $3 \times 10^{-8}$ Torr

${ }^{a)}$ Electronic mail: ig.tsong@asu.edu when the SSJ was in operation. The typical flux rates were $(1-10) \times 10^{13} \mathrm{~cm}^{-2} \mathrm{~s}^{-1}$ for $\mathrm{Ga}$ from an evaporative cell, and $(0.3-3) \times 10^{13} \mathrm{~cm}^{-2} \mathrm{~s}^{-1} \mathrm{NH}_{3}$ from the SSJ. Since the deposition was conducted in situ in the LEEM within the $2 \mathrm{~mm}$ gap between the objective lens and the substrate surface, the necessity to avoid high-voltage breakdowns (which could be caused by accumulation of deposits around the lens as well as high background gas pressure) meant that the upper limits of the quoted fluxes defined the highest rate of film growth. Thermal desorption measurements by Ambacher et al. ${ }^{9}$ show that the onset of $\mathrm{GaN}$ decomposition occurs at $750{ }^{\circ} \mathrm{C}$, a result supported by the maximum deposition rate occurring at $760-780{ }^{\circ} \mathrm{C}$ observed by Lee et al. ${ }^{10}$ in gas-source molecular beam epitaxy (GSMBE) of $\mathrm{GaN}(0001)$ with fluxes of $6 \times 10^{14} \mathrm{~cm}^{-2} \mathrm{~s}^{-1}$ for $\mathrm{Ga}$ and $1 \times 10^{17} \mathrm{~cm}^{-2} \mathrm{~s}^{-1}$ for $\mathrm{NH}_{3}$. Because of the low growth rates in the LEEM, in order to avoid decomposition, we chose to conduct the growth experiments in the temperature range of $665-710^{\circ} \mathrm{C}$.

The substrates used in the present work were $1.4-\mu \mathrm{m}$ thick $\mathrm{GaN}(0001)$ layers grown by metalorganic chemical vapor deposition (MOCVD) on 0.1- $\mu$ m-thick AlN buffer layers on $6 \mathrm{H}-\mathrm{SiC}(0001)$ mounted on a rotating susceptor described previously. ${ }^{11}$ The substrate surface was cleaned in the LEEM by exposure to a flux of $\mathrm{N}$ atoms from an EPI rf-plasma source operated at $200 \mathrm{~W}$ and a nitrogen pressure of $\sim 1$ $\times 10^{-5}$ Torr for $10-15 \mathrm{~min}$ around $675^{\circ} \mathrm{C}$. After the cleaning procedure, the $\mathrm{GaN}$ substrate surface usually displayed a $(\sqrt{3} \times \sqrt{3})$ low-energy electron diffraction (LEED) pattern. Additional treatment by $\mathrm{Ga}$ deposition in the temperature range of $600-730{ }^{\circ} \mathrm{C}$ led to a $(2 \times 2)$ LEED pattern on some of the substrates. The LEEM and LEED operations were interchangeable at any time during the growth experiments simply by inserting or removing the contrast aperture in the conjugate diffraction plane. ${ }^{8}$ Bright-field imaging was conducted throughout, i.e., the (00) LEED beam was selected for imaging.

Figure 1 shows frame-captured LEEM images of $\mathrm{GaN}$ homoepitaxial growth at $677^{\circ} \mathrm{C}$ with the $\mathrm{NH}_{3}$ flux at 0.7 

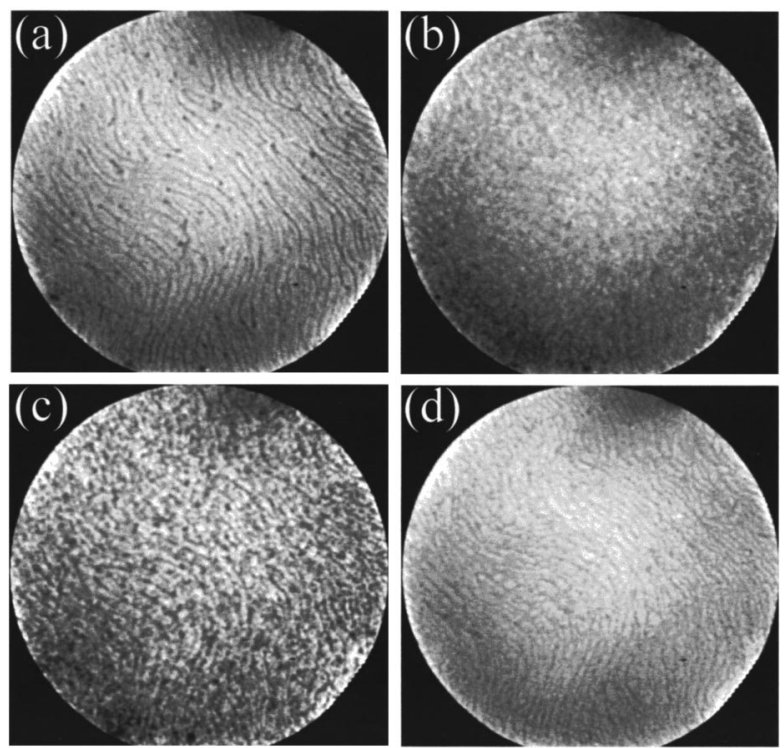

FIG. 1. Frame-captured LEEM video images of homoepitaxial growth of $\mathrm{GaN}$ on a MOCVD GaN $(0001)$ substrate: (a) initial $(\sqrt{3} \times \sqrt{3})+(2 \times 2)$ surface of the GaN substrate; (b) after 6 min of deposition; (c) after 9 min; and (d) after $120 \mathrm{~min}$. Substrate temperature $677^{\circ} \mathrm{C}$. Ga flux 5.9 $\times 10^{13} \mathrm{~cm}^{-2} \mathrm{~s}^{-1} ; \mathrm{NH}_{3}$ flux $0.7 \times 10^{13} \mathrm{~cm}^{-2} \mathrm{~s}^{-1}$. Electron energy $11.0 \mathrm{eV}$. Field of view $4.8 \mu \mathrm{m}$.

$\times 10^{13} \mathrm{~cm}^{-2} \mathrm{~s}^{-1}$ and Ga flux at $5.9 \times 10^{13} \mathrm{~cm}^{-2} \mathrm{~s}^{-1}$, i.e., a $\mathrm{Ga} / \mathrm{NH}_{3}$ flux ratio of $\sim 8$. After 6 min of deposition, the meandering steps of the substrate surface in frame (a) were largely obscured, replaced by a grainy surface morphology in frame (b). After 9 min of deposition, the grainy appearance was still present, but the meandering step structure began to reemerge in frame (c). After about $2 \mathrm{~h}$ of deposition, the meandering steps were restored and the surface showed graininess too fine to be seen in frame (d), but clearly visible in the original images on the LEEM video monitor. The GaN film surface did not change significantly after frame (d), retaining the same appearance even after two more hours of deposition. The LEED pattern of the surface sequence was $(5 \times 5)$ after $20 \mathrm{~min}$, then became $(1 \times 1)$ after $40 \mathrm{~min}$ and throughout the remaining growth. No facet spots were observed.

The growth rate was determined by measuring the film thickness using an atomic force microscope (AFM) after the sample was removed from the LEEM. The thickness was determined by the step height at the boundary between the film and the substrate where no growth had occurred. However, because of the difficulty in distinguishing the film from the substrate in homoepitaxy, we found it impossible to locate such a boundary with the AFM. The growth rate was, therefore, determined, albeit indirectly, from a heteroepitaxy experiment in which a $\mathrm{GaN}$ film was grown on a $6 \mathrm{H}-\mathrm{SiC}$ (0001) substrate using flux rates comparable to those used in Fig. 1. The thickness of the heteroepitaxial film was measured at $\sim 20 \mathrm{~nm}$ for a growth period of $4 \mathrm{~h}$, which translates into a growth rate of $\sim 5 \mathrm{~nm}$ per hour.

We achieved the same growth sequence on a different substrate with the same $\mathrm{Ga} / \mathrm{NH}_{3}$ flux ratio of $\sim 8$, but at half the flux rates as in Fig. 1. The growth temperature was slightly lower at $665^{\circ} \mathrm{C}$. In the LEEM video, while the sequence of development of surface morphology followed the Downloaded 16 Apr 2008 to 152.1.24.180. Redistribution subject

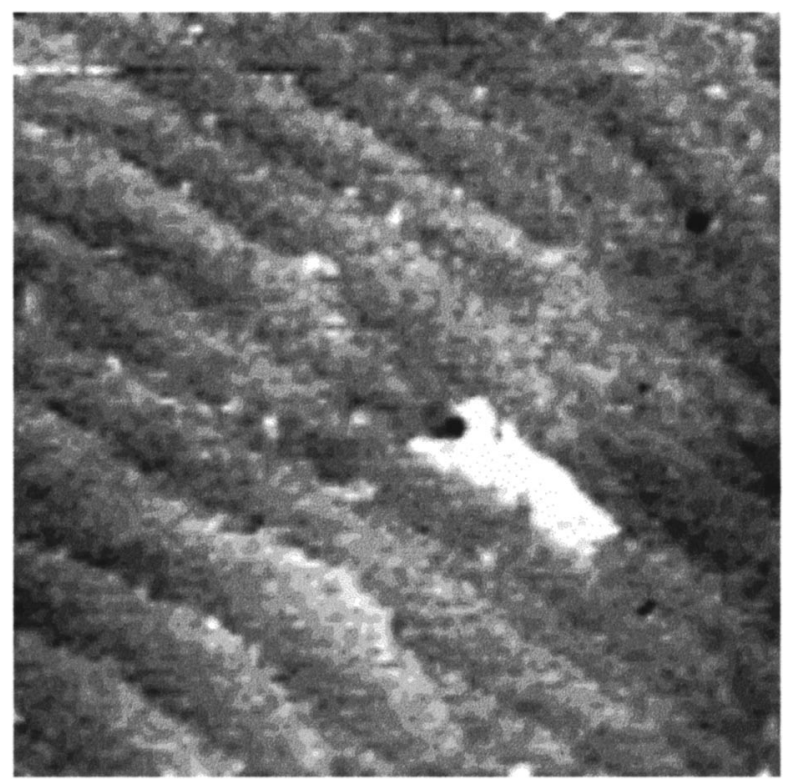

FIG. 2. AFM image of the $\mathrm{GaN}(0001)$ homoepitaxial layer as shown in frame (d) of Fig. 1. Scan area $1 \mu \mathrm{m} \times 1 \mu \mathrm{m}$.

same order as in Fig. 1, the overall time taken to reach the smooth meandering stepped surface similar to frame (d) took longer, $\sim 210 \mathrm{~min}$ instead of $\sim 120 \mathrm{~min}$. This illustrates that while the individual fluxes can be varied, maintaining the flux ratio is essential to achieve (0001) basal-plane growth.

We interpret our in situ real-time LEEM/LEED observations of GaN homoepitaxy as quasi-two-dimensional (2D) island growth similar to the model proposed by Headrick et al. ${ }^{12}$ for GSMBE of cubic GaN of $\beta$-SiC(001). Frame (b) of Fig. 1 represents the initial growth of islands large enough to be resolved by the LEEM. The lateral growth of these islands led to a continuous layer. Once this layer was formed, the meandering steps in frame (d) resumed the sharper appearance as that shown in frame (a) of Fig. 1. Growth continued at a steady rate in islands at the resolution limit of our LEEM. Hence, frame (d) in Fig. 1 appears unchanged over two or more hours of deposition. This conjecture is supported by ex situ AFM images taken on the GaN homoepitaxial layer shown in Fig. 1(d). The AFM image in Fig. 2 shows that the terraces consisted of $\sim 10$-nm-sized grains or islands, in qualitative agreement with the resolution limit of our LEEM. The nucleation of these small islands with the $(0001)$ basal plane as indicated by the $(1 \times 1)$ LEED pattern with a strong (00) spot is probably due to a change in the growth kinetics. The initial nucleation occurred on a chemically modified MOCVD GaN(0001) substrate surface, possibly not completely cleaned even after our cleaning treatment. The initially completed homoepitaxial GaN(0001) layer was of higher purity because it was grown under UHV conditions. The nucleation rate on this pure surface was much higher leading to a high density of very small 2D crystals which coalesced before they could be resolved in the LEEM. Thus, while film growth continued after frame (d), the grainy appearance in frames (b) and (c) of the LEEM images never reappeared.

Our in situ real-time LEEM investigations of GaN homoepitaxy by SSJ leading to basal plane growth are summarized in Fig. 3 where we have plotted the $\mathrm{Ga} / \mathrm{NH}_{3}$ flux ratio AlP license or copyright; see http://apl.aip.org/apl/copyright.jsp 


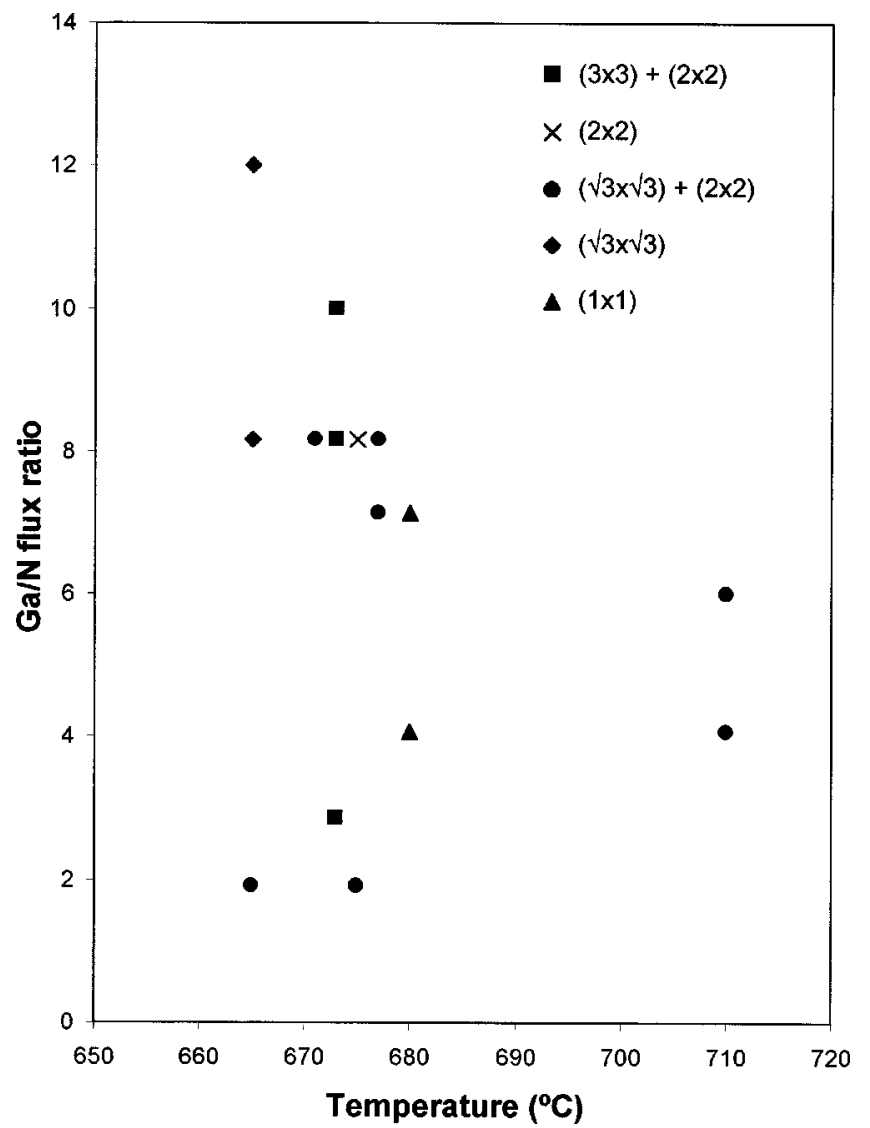

FIG. 3. Plot of $\mathrm{Ga} / \mathrm{NH}_{3}$ flux ratio vs growth temperature illustrating nonfaceted homoepitaxial growth. The ranges of flux rates are: $(1-10)$ $\times 10^{13} \mathrm{~cm}^{-2} \mathrm{~s}^{-1}$ for $\mathrm{Ga}$ and $(0.3-3) \times 10^{13} \mathrm{~cm}^{-2} \mathrm{~s}^{-1}$ for $\mathrm{NH}_{3}$. The different symbols indicate the LEED patterns observed for the substrate surfaces prior to growth.

versus the growth temperature in the range of $665-710^{\circ} \mathrm{C}$. The different symbols in Fig. 3 indicate the LEED patterns observed for the substrates after the $\mathrm{N}$-atom cleaning and Ga-deposition treatment described earlier. It appears that as long as the $\mathrm{Ga} / \mathrm{NH}_{3}$ flux ratio was greater or equal to 2, nonfaceted growth was achieved. We should point out that the term "nonfaceted" growth refers to all forms of (0001) basal-plane growth which do not contain facets, i.e., the LEED pattern shows only $(1 \times 1)$ with a strong $(00)$ LEED spot. However, not all nonfaceted growth exhibited a welldefined step structure such as those shown in frame (d) of Fig. 1. Some of the nonfaceted basal plane layers show a grainy appearance in the LEEM images. AFM measurements of these surfaces do not produce any meaningful step heights between two adjacent grains. When the $\mathrm{Ga} / \mathrm{NH}_{3}$ ratio falls below 2, facet spots appear in the LEED pattern, the (00) spot fades in intensity, and the LEEM image shows a grainy and spotty surface with reduced contrast. The development of faceted growth is easier to follow with LEED rather than LEEM, as we have demonstrated previously. ${ }^{4}$ While we found that we could always convert a nonfaceted $\mathrm{GaN}$ layer into a faceted layer simply by lowering the $\mathrm{Ga} / \mathrm{NH}_{3}$ flux ratio to $<2$, we could not convert a well-developed faceted layer back into basal plane growth by increasing the flux ratio. This points to the fact that the formation of facets on the GaN film surface is thermodynamically preferable, but basalplane growth could be achieved and maintained by carefully controlling the kinetic factors.

In summary, we have achieved smooth basal-plane layer growth in $\mathrm{GaN}(0001)$ homoepitaxy using a $\mathrm{NH}_{3}$ seededbeam SSJ at a growth temperature range of $665-710^{\circ} \mathrm{C}$. In situ real-time LEEM combined with LEED is a powerful method for the study of growth processes because the instant feedback it provides allows changing of parameters to optimize growth during observation.

The authors thank Uwe Knipping and John Edwards for their technical assistance. This work was supported by the Office of Naval Research, Grant No. N00014-95-1-0122, and also in part by NSF-MRSEC Grant No. DMR-9632635.

${ }^{1}$ S. T. Ceyer, Science 249, 133 (1990).

${ }^{2}$ S. T. Ceyer, Langmuir 6, 82 (1990).

${ }^{3}$ V. M. Torres, M. Stevens, J. L. Edwards, D. J. Smith, R. B. Doak, and I. S. T. Tsong, Appl. Phys. Lett. 71, 1365 (1997).

${ }^{4}$ A. Pavlovska, E. Bauer, V. M. Torres, J. L. Edwards, R. B. Doak, I. S. T. Tsong, V. Ramachandran, and R. M. Feenstra, J. Cryst. Growth 189/190, 310 (1998).

${ }^{5}$ V. M. Torres, R. B. Doak, B. J. Wilkens, D. J. Smith, and I. S. T. Tsong, J. Vac. Sci. Technol. A 17, 1570 (1999).

${ }^{6}$ J. Fritsch, O. F. Sankey, K. E. Schmidt, and J. B. Page, Surf. Sci. 427/428, 298 (1999).

${ }^{7}$ B. A. Ferguson and C. B. Mullins, J. Cryst. Growth 178, 134 (1997).

${ }^{8}$ E. Bauer, Rep. Prog. Phys. 57, 895 (1994); R. M. Tromp and M. C. Reuter, Ultramicroscopy 50, 171 (1993).

${ }^{9}$ O. Ambacher, M. S. Brandt, R. Dimitrov, T. Metzger, M. Stutzmann, R. A. Fischer, A. Miehr, A. Bergmaier, and G. Dollinger, J. Vac. Sci. Technol. B 14, 3532 (1996).

${ }^{10}$ N. E. Lee, R. C. Powell, Y. W. Kim, and J. E. Greene, J. Vac. Sci. Technol. A 13, 2293 (1995).

${ }^{11}$ T. W. Weeks, Jr., M. D. Bremser, K. S. Ailey, E. P. Carlson, W. G. Perry, and R. F. Davis, Appl. Phys. Lett. 67, 401 (1995).

${ }^{12}$ R. L. Headrick, S. Kycia, Y. K. Park, A. R. Woll, and J. D. Brock, Phys. Rev. B 54, 14686 (1996). 